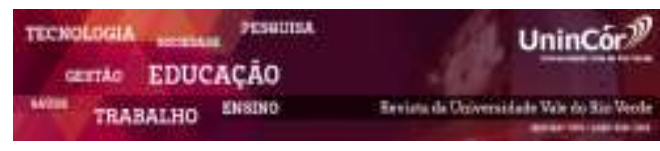

Revista da Universidade Vale do Rio Verde ISSN: 1517-0276 / EISSN: 2236-5362 v. 16 | n. 2 | Ano 2018

Lilian Keylla Berto Centro Universitário de Maringá lilianberto_engenharia@hotmail.com

Renan Godoi

Centro Universitário de Maringá rnngodoi@gmail.com

Lídia Pereira Amaro

Centro Universitário de Maringá lidia_amaro@hotmail.com

Marla Corso

Centro Universitário de Maringá marlacorso@ hotmail.com

Judson Ricardo Ribeiro

Centro Universitário de Maringá judson@unicesumar.edu.br

Luciana Cristina Soto Herek Rezende Centro Universitário de Maringá luciana.rezende@unicesumar.edu.br

\section{ANÁLISE DA RELAÇÃ̃ ENTRE A PERMEABILIDADE E AS CARACTERÍSTICAS MECÂNICAS DO REVESTIMENTO ASFÁLTICO PERMEÁVEL}

\section{RESUMO}

$\mathrm{O}$ avanço industrial e social geram impactos ambientais sendo essencial a busca por meios sustentáveis. Este artigo teve por finalidade a utilização do asfalto permeável como objetivo de avaliar por ensaios mecânicos e hidráulicos três modelos de revestimento asfáltico de pavimentos permeáveis, em comparação com um pavimento convencional. Para a realização dos ensaios foram moldados corpos de prova submetidos aos ensaios de desgaste por abrasão (DNER-ME 383/1999), resistência a tração por compressão diametral (DNER-ME 138/1994) e permeabilidade (NLT-327/00).Os resultados obtidos mostram que a quantidade de vazios é diretamente proporcional à permeabilidade e ao desgaste por abrasão, e inversamente proporcional a resistência à tração por compressão diametral.Assim, tem-se que quanto maior o índice de vazios maior a permeabilidade do material e devido à elevada porcentagem de vazios os valores de resistência à tração diminuíram e o desgaste por abrasão aumentou.Dentre os modelos a "Faixa III" atendeu as normativas aplicadas sendo recomendada para tráfego leve, a fim de garantir conforto e segurança aos usuários, resultando em uma redução de impacto ambiental.

Palavras-chave: Sustentabilidade. Pavimento permeável. Ensaios hidráulicos. Revestimento Asfáltico. Faixas granulométricas.

\section{ANALYSIS OF RELATIONSHIP BETWEEN PERMEABILIDAE AND MECHANICAL FINISHING ASPHALT PERMEABLE FEATURES}


standards applied being recommended for light traffic, in order to guarantee comfort and safety to users, resulting in a reduction of environmental impact.

Keywords: Sustainability. Permeable floor. Hydraulic tests. Asphalt Coating. Granulometric bands.

\section{Recebido em: 30/07/2018 - Aprovado em: 09/12/2018 - Disponibilizado em: 15/12/2018}

\section{INTRODUÇÃO}

Em virtude do acelerado crescimento urbano e industrial, uma das necessidades atuais, são os meios de transportes.Um exemplo comum é a malha rodoviáriaque geralmente utiliza-se dopavimento convencional. Este últimoé apontado por ser um dos principais causadores de transtorno e desconforto aos usuários, devido sua característica impermeávelque permite exceder a capacidade dos sistemas de drenagem, tornandoos incapazes na ocorrência de chuvas intensas, aumentando os riscos de alagamentos e enchentes. O fator agravante são os aumentos decontaminantes e sedimentos no escoamento pluvial que prejudicam a qualidade da água nos córregos e rios. (HU, et al, 2018; THIVES, et al, 2018).

Por consequência, a forma vigente empregada para o desenvolvimento urbano gera impactos negativos ao meio ambiente, tanto dos veículos que emitem ao ambiente compostos orgânicos voláteis, como também, a falta de maneiras eficientes para combater as poluições, resultando no transporte de poluentes para o solo e água, além de aumentar o desconforto térmico devido ao alto calor liberado pela superfície (HUANG; LIANG, 2017; WU, et al., 2018). Um dos meios sustentáveis é a aplicação do asfalto permeável, visando à manutenção eficiente a fim debeneficiar o meio ambiente e a sociedade através deste sistema (VALEO; GUPTA, 2018).
O pavimento asfáltico permeável tem a função de infiltrar a água proveniente das chuvas através de camadas que constituem o sistema (THIVES, et al, 2018). Uma das suas maiores características é a alta porosidade na camada de revestimento. $\mathrm{O}$ asfalto poroso é uma mistura com alto teor de vazios, dependo do tipo de agregado e da quantidade de ligante asfáltico que será utilizado na mistura, o índice de vazios pode chegar a valores superiores a 20\% (VALERI, et al, 2018).

Para alcançar a permeabilidade desejada, existem fatores que influenciam, como a granulometria dos agregados, a porcentagem de vazios, a natureza do ligante e a espessura do revestimento. Podem também ser acrescentados ao concreto betuminoso usinado a quente (CBUQ) aditivos ou resíduos como uso de cinzas de carvão, materiais fibrosos, entre outros(KOLODZIEJ, 2016; RAD; MODARRES, 2017). Convencionalmente, para a produção de um asfalto permeável, utiliza-se uma granulometria aceitável pela norma DNIT 031/2006, sendo obtidas através de faixas ligadas diretamente a porcentagem de ligantes.

Para que seja viável a utilização do asfalto permeável é necessário que o mesmo atenda parâmetros de resistência à tração e ao desgaste, como também deve ser analisado o comportamento da permeabilidade, a fim de ser eficiente, mantendo o controle de qualidade exigido por norma. Outra característica 
primordial a ser levada em consideração é a porcentagem de vazios determinadas em relação ao volume do asfalto (FERREIRA, 2015).

Diante do exposto, o presente trabalho teve por objetivo aavaliação mecânica e hidráulica de um pavimento asfáltico utilizando diferentes granulometrias de agregados, visando desta forma soluções quanto à necessidade de otimizar o escoamento da água em sistemas de drenagem.

\section{MÉTODOS}

\subsection{Preparação da Mistura}

Os agregados graúdos utilizados são de origem mineral e provindos da britagem de rochas basálticas. Foram coletadas as amostras e elaboradas quatro faixas granulométricas diferentes com variação do diâmetro dos agregados. Para realização da separação granulométrica os agregados foram submetidos ao conjunto de peneiras com agitação mecânica, conforme a norma DNER-ME 080/1994. O ligante utilizado para a realização dos ensaios com o revestimento de material asfáltico o CAP 50/70 fornecido pela empresa de pavimentação Conterpavi Construções LTDA, situada em Maringá, PR.

\subsection{Produção dos Corpos de Prova}

Foram moldados quarenta e oito corpos de prova para determinação de índice de vazios, permeabilidade, resistência à tração e ao desgaste, sendo doze para cada faixa granulométrica. Foram determinadas quatro faixas granulométricas. Na qual a "Faixa C", conforme DNER-ES 031/2006 e a "Faixa III", Camada Porosa de Atrito - CPA, de acordo com DNER-ES 389/1998 foramdotadas de um pavimento convencional, o concreto betuminoso usinado a quente (CBUQ). Os demais seguiram o modelo de revestimento alfáltico permeável, conforme a revisão literária considerando as especificações. Dessa forma a "Faixa Ref.1", em conformidade com os estudos de Azzout et al (1994) e a "Faixa Ref. 2" adota segundo estudos de Acioli (2005).

O teor do ligante é de 5,0\% para a "Faixa C". As demais faixas granulométricas com o teor de ligante de $4,6 \%$.

Para a moldagem dos corpos de prova os agregados e o ligante foram aquecidos em estufa separadamente à temperatura entre $105^{\circ} \mathrm{C}$ a 110 ${ }^{\circ} \mathrm{C}$. Em seguida os recipientes utilizados foram conduzidos a uma estufa para aquecer a uma temperatura de $150{ }^{\circ} \mathrm{C}$. Ato contínuo passou-se com a mistura dos agregados e o ligante em cada recipiente de acordo com suas proporções até a cobertura completa dos agregados.

A mistura foi adicionada ao molde, e sobre ela foi colocado um filtro para se proceder à compactação das amostras com auxílio de um soquete foram aplicados 75 (setenta e cinco) golpes na face inferior e superior da mistura, para se modelar o corpo de prova da "Faixa C". Para a CPA "Faixa III", "Faixa Ref. 1" e "Faixa Ref. 2" foram 50 (cinquenta) golpes, conforme especificação da norma DNER-ES 386, visto que a energia de compactação afeta na porcentagem de vazios da amostra.

Os corpos de prova não foram submetidos ao ensaio de estabilidade e fluência (procedimento padrão da norma DNER-ME 043) 
visto que não há especificações de valores destes ensaios, tanto para a CPA quanto para as outras misturas com elevada porcentagem de vazios.

2.3 Determinação do volume de vazios

O material foi pesado em duas condições: ao ar livre e imerso em água (com o auxílio de uma balança hidrostática). Após a imersão, o corpo de prova foi colocado em uma estufa para secar com finalidade de determinar o peso seco. O volume foi definido através da diferença entre o peso seco e o peso imerso. Com o volume conhecido determina-se a densidade aparente através da multiplicação direta do peso ao ar livre e o volume.

O materialfoi aquecido em estufa a $150^{\circ}$ $\mathrm{C}$, por uma hora. Após a retirada da estufa o material foi destorroado, obtendo-se a mesma mistura que se tinha antes da compactação. A mistura foi adicionada ao frasco e então adicionou água até que toda a mistura foi completamente coberta. $\mathrm{O}$ frasco por sua vez foi submetido a uma mesa agitadora onde foi aplicada uma pressão no vácuo por 15 minutos. $\mathrm{O}$ conjunto foi imerso em um banho térmico e posteriormente pesado.

A densidade máxima teórica é determinada pela equação 01 :

$$
\text { Dmt }=\frac{\text { pm }}{\text { pm+pf }- \text { pf m }}
$$

Onde:

Dmt $=$ Densidade máxima teórica

$\mathbf{P m}=$ peso da mistura;

$\mathbf{P f}=$ peso do frasco sem a mistura;

$\mathbf{P f m}=$ peso do frasco com a mistura.

Por fim, definiu-se a porcentagem de vazios através da relação entra a diferença da densidade máxima teórica com a densidade aparente e a densidade máxima teórica.

\subsection{Desgaste por Abrasão}

O ensaio de abrasão ou ensaio de cantabro foi de acordo com a norma DNER-ME 383/99 onde após moldado os corpos de prova estes foram levados a um aparelho denominado máquina Los Angeles para obter o desgaste.

$\mathrm{O}$ material foi pesado e anotado o seu peso. Logo após o corpo de prova foi colocado no tambor de ensaio sem adicionar as cargas abrasivas que compõem o aparelho. Após ligar a máquina o copo de prova foi submetido a uma velocidade periférica uniforme em torno de 30 a $33 \mathrm{rpm}$, até o tambor completar 300 rotações. Por fim retira-se o material do tambor e anota-se o seu peso.

Determinou-se a porcentagem do desgaste, sendo a relação da diferença do peso total (final e inicial) e o peso total da amostra inicial.

\subsection{Resistência à Tração por Compressão Diametral}

A resistência à tração por compressão diametral sucedeu-se de acordo com a norma DNER-ME 138/94. Iniciou-se com a coleta de informações como diâmetro médio das amostras. Em seguida, a execução do ensaio as amostras estavamem temperatura de $45{ }^{\circ} \mathrm{C}$. Foi utilizada uma prensa mecânica com o mesmo equipamento do ensaio Marshall, mas com frisos metálicos que impõem as cargas de tração.

As amostras foram colocadas sobre o prato inferior da prensa e então foram ajustados 
os pratos da prensa até uma leve compressão capaz de manter em posição o corpo de prova. Por fim a carga foi aplicada até a ruptura do corpo de prova. Com os valores de carga de ruptura obtidos, a resistência à tração por compressão diametral é calculada através da equação 02:

$$
\operatorname{Tr}=\frac{2 F}{100 \pi \text { D H }} \text { Eq. } 02
$$

Onde:

$\mathbf{T r}=$ Tensão de ruptura $(\mathrm{MPa}) ;$

$\mathbf{F}=$ Força de ruptura $(\mathrm{MPa})$;

$\mathbf{D}=$ Diâmetro da amostra $(\mathrm{cm})$;

$\mathbf{H}=$ Altura da amostra $(\mathrm{cm})$.

2.6Permeabilidade

O ensaio de permeabilidade foi realizado conforme o estudo realizado por Nascimento (2012), utilizando como referência a norma espanhola NLT-327/00, no qual foi desenvolvido um permeâmetro de carga constante para ensaios em laboratórios.

O instrumento de realização do ensaio consiste em um equipamento de carga constante, sendo um tubo de PVC com altura de $60 \mathrm{~cm}$. Este tubo possui um dreno a $50 \mathrm{~cm}$ de altura (altura da coluna d'água para cálculos) para a água manter o nível da coluna. Sob o tubo fica o corpo de prova revestido com uma manta de borracha para que a água não escoe pela parede lateral do corpo de prova. O ensaio foi realizado a uma temperatura ambiente de $25^{\circ} \mathrm{C}$. A coluna d'água foi mantida durante 10 (dez) min. Em seguida foi inserido um recipiente, de peso conhecido, abaixo do equipamento para coletar a quantidade de água que passa pelo corpo de prova durante um minuto.
O recipiente foi pesado e então calculado a vazão pela equação 03 :

$$
Q=\left(\frac{m 2-m 1}{t}\right)^{x 10-6}
$$

Onde:

$\mathbf{Q}=\operatorname{Vazão}\left(\mathrm{m}^{3} / \mathrm{s}\right)$

$\mathbf{m} \mathbf{2}=$ massa do recipiente com líquido após o ensaio $(\mathrm{g})$

$\mathbf{m 1}=$ massa do recipiente antes do ensaio $(\mathrm{g})$

$\mathbf{t}=$ tempo de aplicação do ensaio (s)

Após conhecido a vazão o coeficiente de permeabilidade é calculado pela equação 04:

$$
\mathbf{K}=\frac{\mathrm{QL}}{\mathrm{AH}}
$$

Onde:

$\mathbf{K}=$ Coeficiente de permeabilidade $(\mathrm{m} / \mathrm{s})$;

$\mathbf{Q}=$ vazão de entrada $\left(\mathrm{m}^{3} / \mathrm{s}\right)$;

$\mathbf{L}=$ altura do corpo de prova (m);

$\mathbf{A}=$ área da seção transversal do corpo de prova $\left(\mathrm{m}^{2}\right)$

$\mathbf{H}=$ Altura da coluna d'água correspondente à pressão utilizada $(\mathrm{cm})$.

\section{RESULTADOS E DISCUSSÃO}

As análises granulométricas são dispostas em variação do diâmetro dos agregados. O resultado da disposição de peneiras, segundo a norma DNER-ME 080/1994 é expressa em porcentagem na Tabela 1 , que revela a quantidade em percentual dos agregados que estão retidos, acumulados epassando em cada peneira. Esse ensaio foi realizado para faixas granulométricas, sendo elas: "Faixa C", "Faixa III", "Faixa Ref.1" e "Faixa Ref.2". 
Tabela 1 -Análises granulométricas segundo os itens 7.5; 7.6 e 7.7 da norma DNER-ME 080/1994

\begin{tabular}{|c|c|c|c|c|c|c|c|c|c|c|c|}
\hline \multicolumn{12}{|c|}{$\begin{array}{l}7.5 \text { - \% AMOSTRA TOTAL SECA RETIDA EM CADA PENEIRA; } 7.6 \text { - \% MATERIAL ACUMULADO } \\
\text { SECO EM CADA PENEIRA; } 7.7 \text { - \% MATERIAL SECO PASSANDO EM CADA PENEIRA }\end{array}$} \\
\hline PENEIR & & & $1 "$ & 3/4" & $1 / 2^{\prime \prime}$ & $3 / 8 "$ & $\mathrm{~N}^{\circ} 4$ & $\mathrm{~N}^{\mathrm{o}} 10$ & $\mathrm{~N}^{\circ} 40$ & $\mathrm{~N}^{\circ} 80$ & $\mathrm{~N}^{\mathrm{o}} 200$ \\
\hline \multirow{3}{*}{$\begin{array}{l}\text { "FAIXA } \\
\text { C" }\end{array}$} & & & & $0,00 \%$ & $12,35 \%$ & & & & & & $7,51 \%$ \\
\hline & 7.6 & $0,00 \%$ & $0,00 \%$ & $0,00 \%$ & $12,35 \%$ & $20,16 \%$ & $40,25 \%$ & $58,00 \%$ & $76,11 \%$ & $86,09 \%$ & $93,60 \%$ \\
\hline & 7.7 & $100,00 \%$ & $100,00 \%$ & $100,00 \%$ & $87,65 \%$ & $79,84 \%$ & $59,75 \%$ & $42,00 \%$ & $23,89 \%$ & $13,91 \%$ & $6,40 \%$ \\
\hline \multirow{3}{*}{$\begin{array}{l}\text { "FAIXA } \\
\text { III" }\end{array}$} & 7.5 & $0,00 \%$ & $0,00 \%$ & $0,00 \%$ & $0,00 \%$ & $13,32 \%$ & $39,88 \%$ & $30,76 \%$ & $7,71 \%$ & $0,00 \%$ & $3,07 \%$ \\
\hline & 7.6 & $0,00 \%$ & $0,00 \%$ & $0,00 \%$ & $0,00 \%$ & $13,32 \%$ & $53,20 \%$ & $83,96 \%$ & $91,67 \%$ & $91,67 \%$ & $94,74 \%$ \\
\hline & 7.7 & $100,00 \%$ & $100,00 \%$ & $100,00 \%$ & $100,00 \%$ & $86,68 \%$ & $46,80 \%$ & $16,04 \%$ & $8,33 \%$ & $8,33 \%$ & $5,26 \%$ \\
\hline \multirow{3}{*}{$\begin{array}{l}\text { "FAIXA } \\
\text { REF. 1" }\end{array}$} & 7.5 & $0,00 \%$ & $0,00 \%$ & $0,00 \%$ & & $8,88 \%$ & $53,04 \%$ & $25,55 \%$ & $5,13 \%$ & $0,00 \%$ & $3,55 \%$ \\
\hline & 7.6 & $0,00 \%$ & $0,00 \%$ & & & & & & $92,60 \%$ & $92,60 \%$ & $96,15 \%$ \\
\hline & 7.7 & $100,00 \%$ & $100,00 \%$ & $100,00 \%$ & $100,00 \%$ & $91,12 \%$ & $38,07 \%$ & $12,52 \%$ & $7,40 \%$ & $7,40 \%$ & $3,85 \%$ \\
\hline \multirow{3}{*}{$\begin{array}{l}\text { "FAIXA } \\
\text { REF.2" }\end{array}$} & 7.5 & $0,00 \%$ & $0,00 \%$ & $0,00 \%$ & $0,00 \%$ & $0,00 \%$ & $22,85 \%$ & $59,00 \%$ & $12,26 \%$ & $0,00 \%$ & $0,00 \%$ \\
\hline & 7.6 & $0,00 \%$ & $0,00 \%$ & $0,00 \%$ & $0,00 \%$ & $0,00 \%$ & $22,85 \%$ & $81,85 \%$ & $94,11 \%$ & $94,11 \%$ & $94,11 \%$ \\
\hline & 7.7 & $100,00 \%$ & $100,00 \%$ & $100,00 \%$ & $100,00 \%$ & $100,00 \%$ & $77,15 \%$ & $18,15 \%$ & $5,89 \%$ & $5,89 \%$ & $5,89 \%$ \\
\hline
\end{tabular}

Fonte:Autores.

A partir dos resultados das análises granulométricas foi possível identificar a influência dos diâmetros dos agregados no revestimento asfáltico.Mecanicamente, para um tráfego mais pesado e intenso, maior será o tamanho do agregado. E para um pavimento mais rígido, menor será este tamanho do agregado.O objetivo alcançado foi que as faixas apresentaram distribuição granulométrica contínua,ou seja, agregados de graduação densa ou bem-graduada.

O índice de vazios, a permeabilidade, a resistência à tração e ao desgaste por abrasão foram os demais ensaios, sendo que cada teste utilizou três corpos de provas e a média dos resultados está contida naFigura 1.

Houve variações de permeabilidade nos corpos de provas consideráveis. Os três corpos de prova da "Faixa Ref. 2" mostraram valores $(2,00$ $\mathrm{mm} / \mathrm{s} ; 1,95 \mathrm{~mm} / \mathrm{s}$ e $1,93 \mathrm{~mm} / \mathrm{s}$ ) bem próximos os encontrados por Nascimento (2012). Outras duas faixas contendo material asfáltico permeável são a "Faixa III" que mostrou os corpos de provas com valores destacáveis $(1,05 \mathrm{~mm} / \mathrm{s} ; 1,02 \mathrm{~mm} / \mathrm{s}$; $0,987 \mathrm{~mm} / \mathrm{s}$ ) que ficaram bem próximos aos valores obtidos por Silva (2005) que eram de $1,05 \mathrm{~mm} / \mathrm{s}$. A“Faixa Ref. 1" apresentou os três corpos de provas com desempenho satisfatórios de permeabilidade $\left(1,66 \times 10^{-3} \mathrm{~mm} / \mathrm{s} ; 1,63 \times 10^{-3}\right.$ $\mathrm{mm} / \mathrm{s}$ e $1,60 \times 10^{-3} \mathrm{~mm} / \mathrm{s}$ ). A“Faixa C" não pode ser considerada permeável, devido seu baixo desempenho hidráulico, os resultados foram de $0,208 \mathrm{~mm} / \mathrm{s} ; 0,204 \mathrm{~mm} / \mathrm{s}$ e $0,209 \mathrm{~mm} / \mathrm{s}$. A norma europeia EN 12697-19 (2004) estabelece valores de permeabilidade. Segundo esta norma são considerados revestimentos permeáveis aqueles que atingirem a permeabilidade entre $0,5 \mathrm{~mm} / \mathrm{s}$ a $3,5 \mathrm{~mm} / \mathrm{s}$.

A resistência por desgaste por abrasão apresentou bons resultados na "Faixa C", "Faixa III" e "Faixa Ref. 2". Visto que o desgaste máximo deveria ser de 25\%, segundo DNER-ES 386/1999, de acordo com os resultados apresentados naFigura 1, o material da "Faixa Ref.1" ultrapassou o limite da norma. 
A resistência à tração mínima especificada pela norma DNER-ES 386/1999 é de 0,55 MPa. Por este motivo, os resultados mostrados no Gráfico 1, comprovam que os materiais da "Faixa Ref.1" e "Faixa Ref.2" não atingiram o mínimo de resistência especificado na norma.

Pode-se observar que o material para ser considerado permeável, é fundamental possuir um índice de vazios maior que $10 \%$. É o que explica o baixo desempenho drenante na "Faixa C". Esta informação nos mostra que quanto maior a porcentagens de vazios do material maior será a permeabilidade. Em valores aproximados, uma comparação de material com $20 \%$ de vazios e outro com $40 \%$, esse ultimo apresenta permeabilidade duas vezes maior em relação ao material com índice de vazios de $20 \%$.

Vale ressaltar que os resultados de resistência à tração por compressão diametral diminuíram conforme o aumento da permeabilidade e quanto maior a porcentagem de vazios maior será o desgaste do material.

Figura 1 -Média dos ensaios de índice de vazios, resistência à tração, desgaste por abrasão e permeabilidade

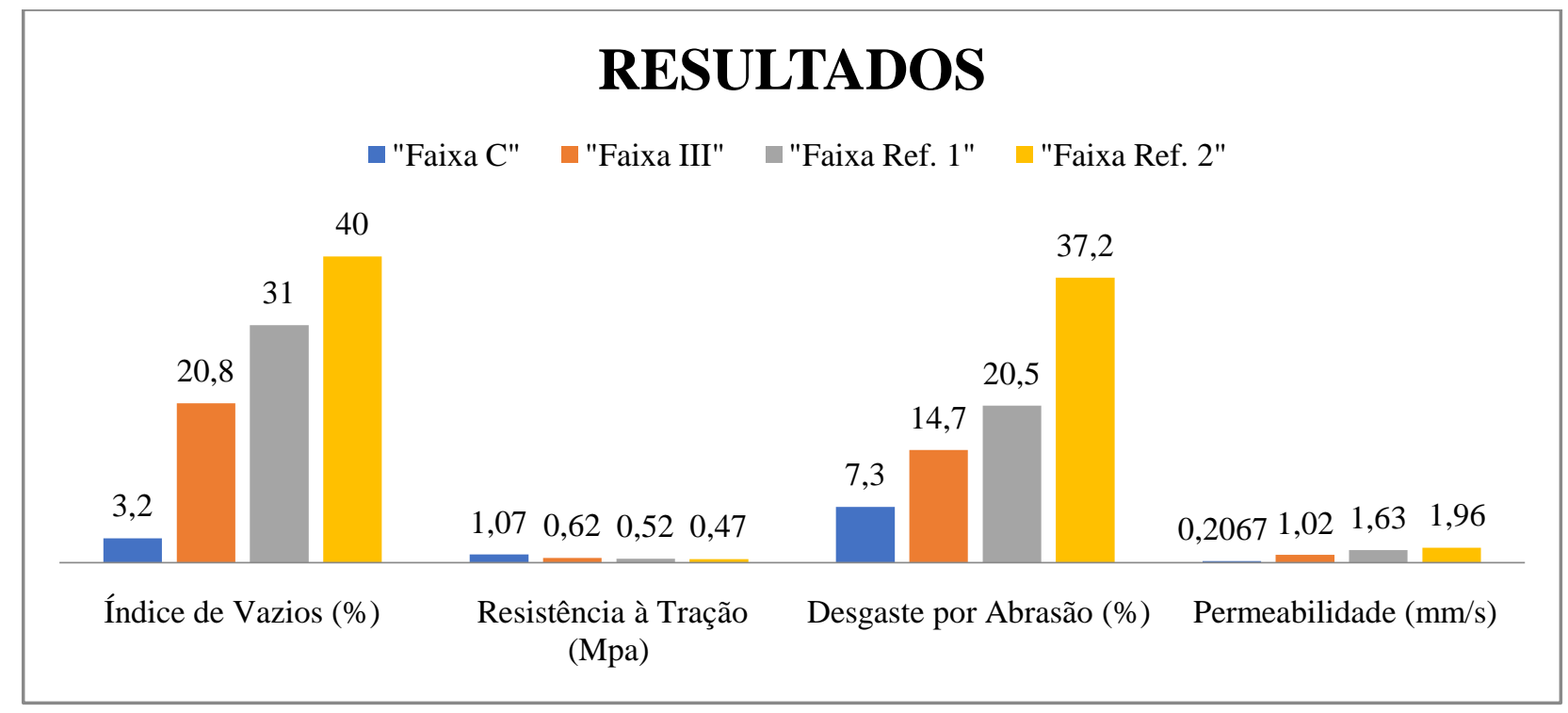

Fonte:Autores.

\section{CONCLUSÃO}

Os resultados mostraram que a permeabilidade é um parâmetro em função do volume de vazios, ou seja, quanto maior volume de vazios maior a permeabilidade do revestimento, logo, devido ao elevado volume de vazios menor será a resistência à tração por compressão diametral e maior será o desgaste por abrasão, parâmetros estes comparados a um pavimento convencional.

Os resultados obtidospara o parâmetro permeabilidade foram bons, com exceção a "Faixa C", sendoa redução de vazios o fator que mais influenciou este resultado insatisfatório. Isso ocorreudevido a sua compactação ter sido a maior em relação aos demais revestimentos. A DNER-ME 43/95, diz que a compactação de 75 golpes aplicada na "Faixa C",resulta em um revestimento que suporta um esforço de pressão de pneu até duas vezes mais em comparação a 
uma compactação de 50 golpes. Embora a "Faixa C" apresentou o melhor desempenho mecânico,porém oseu desempenho hidráulico foi insatisfatório e não pode ser considerado revestimento permeável.

A "Faixa Ref. 1" apresentou o pior resultado mecânico, pois o mínimo de resistência à tração e o máximo ao desgaste por abrasão especificado na norma não foram atingidos.A "Faixa Ref. 2" não teve resultados satisfatórios de resistência à tração. A "Faixa III" foi o melhor resultado tanto mecanicamente quanto de permeabilidade, podendo ser aplicávelcomo revestimento asfáltico em estacionamentos e tráfegos leves,reduzindo significativamente os transtornos causados por alagamentos, aquaplanagem, enchentes, contaminação dos solos e outros.

Assim, torna-se essencial os estudos das propriedades físicas, químicas e mecânicas dos pavimentos para atender as normas, a sustentabilidade, o conforto, a segurança e manter um padrão de qualidade do tráfego para os usuários.

\section{REFERÊNCIAS}

ACIOLI, Laura Albuquerque. Estudo experimental de pavimentos permeáveis para o controle de escoamento superficial na fonte. 2005. 162 f. Dissertação (Mestrado) - Curso de Engenharia, UFRGS, Porto Alegre, 2005.

AZZOUT, Yolande; BARRAUD, Sylvie; CRES, François Noël; ALFAKIH, Elham. Tecniques alternatives en asseinissement pluvial. Choix, conception, réalisation et entretien. Ed. Tec et Doc. Paris: Lavoisier, 1994. 372 p.

CEDEX. NLT - 327. Permeabilidad in situ de pavimentos drenantes com el permeámetro LCS. CEDEX, 2000.
DEPARTAMENTO NACIONAL DE ESTRADAS DE RODAGEM. DNER - ES 386:Pré-misturado a quente: camada porosa de atrito. Rio de Janeiro: DNER, 1999. 15 p.

\section{DEPARTAMENTO NACIONAL DE} ESTRADAS DE RODAGEM. DNER - ES 389/99: Pavimentação - micro revestimento asfáltico a frio com emulsão modificada por polímero. Rio de Janeiro: DNER, 1999. 11p.

\section{DEPARTAMENTO NACIONAL DE ESTRADAS DE RODAGEM. DNER - ME 043: Misturas betuminosas a quente - Ensaio Marshall. Rio de Janeiro: DNER, 1995. 11 p.}

DEPARTAMENTO NACIONAL DE ESTRADAS DE RODAGEM. DNER - ME 138/94: Misturas betuminosas - determinação da resistência a tração por compressão diametral - método de ensaio. Rio de Janeiro: DNER, 1994. 16p.

DNER. DEPARTAMENTO NACIONAL DE
ESTRADAS DE RODAGEM. DNER-ME
080/94: Solos - análise granulométrica por
peneiramento. Rio de Janeiro: DNER, 1994. 4p.

DNER. DEPARTAMENTO NACIONAL DE ESTRADAS DE RODAGEM. DNER-ME 383/99: Desgaste por abrasão de misturas betuminosas com asfalto polímero - ensaio Cantabro. Rio de Janeiro: DNER, 1999. 2p.

DEPARTAMENTO NACIONAL DE INFRAESTRUTURA DE TRANSPORTE. IPR DNIT-ES 031: Pavimentos flexíveis - concreto asfaltico - especificação de serviço.Rio de Janeiro: DNIT, 2006.

\section{EN 12697-19 (2004). Bituminous mixtures - \\ Test methods for hot mix asphalt - Part 19: Permeability of specimen or Marshall specimens.CEN, Brussels.}

FERREIRA, Wellington Lorran Gaia. Análise do fluxo d'água em revestimentos asfálticos com diferentes permeabilidades. 2015. 132f. Dissertação (mestrado em Engenharia de Transportes) - Universidade Federal do Ceará Fortaleza, CE, 2015.

HU, Maochuan; ZHANG Xingqi; SIU Yim Ling; LI Yu; TANAKA Kenji; YANG Hong; XU, Youpeng. Flood mitigation by permeable pavements in chinese sponge city construction. 
Journal Water, [s.1.], v. 172, 12 pages, Fev. 2018. MDPI. https://doi.org/10.3390/w10020172

HUANG, Shengyi; LIANG, Chenju. A conceptual study on the formulation of a permeable reactive pavement with activated carbon additives for controlling the fate of nonpoint source environmental organic contaminants. Chemosphere. [s.1.], v. 193, pages 438-446, 2018.Elsevier. https://doi.org/10.1016/j.chemosphere.2017.11.0 28

KOLODZIEJ, Vanesa María. Estudo das propriedades acústicas, drenantes e mecânicas de revestimentos asfálticos porosos. 2016. 184f. Dissertação (mestrado em Engenharia civil) - Universidade Federal de Santa Catarina Florianópolis, SC, 2016.

NASCIMENTO, Cátia Cristiana Carmona do. Avaliação da permeabilidade de misturas betuminosas drenantes em laboratório e "in situ". Dissertação de mestrado, FEUP, Faculdade de Engenharia da Universidade do Porto, Porto, 2012.

RAD, Seyed Alireza Mohammadi; MODARRES, Amir. Durability properties of non-air entrained roller compacted concrete pavement containing coal waste ash in presence of de-icing salts. Cold Regions Science and Technology, [s.1.], v. 137, pages 48-59, Fev. 2017. Elsevier.

http://dx.doi.org/10.1016/j.coldregions.2017.02.0 06

SILVA, Carlos Eduardo Del Ben Dias da. Estudo da permeabilidade de misturas asfálticas de graduação aberta. Dissertação de mestrado, UNICAMP, Universidade estadual de Campinas, Campinas, 2005.

THIVES, Liseane Padilha; GHISI, Enedir; BRECHT, Douglas Gherardt; PIRES, Dario Menegasso. Filtering capability of porous asphalt pavements. Journal Water, [s.1.], v. 206, 17 pages, Fev.2018. MDPI.

https://doi.org/10.3390/w10020206

VALEO, Caterina; GUPTA, Rishi. Determining surface infiltration rate of permeable pavements with digital imaging. Journal Water, [s.l.], v.133, 22 pages, Jan. 2018. MDPI. https://doi.org/10.3390/w10020133
VALERI, Valerio C. Andrés; TORRES, Javier Rodriguez; PEREZ, Miguel A. Calzada; HERNANDEZ, Jorge Rodriguez. Exploratory study of porous asphalt mixtures with additions of reclaimed tetra pak material. Construction and Building Materials, [s.l.], v. 160, pages 233-239, 2018.

Elsevier.https://doi.org/10.1016/j.conbuildmat.20 17.11.067

WU, Hao; SUN Beibei, LI Zhe; YU, Jia. Characterizing thermal behaviors of various pavement materials and their thermal impacts on ambient environment. Journal of Cleaner Production, [s.1.], v. 172, pages 1358-1367, 2018.

Elsevier.https://doi.org/10.1016/j.jclepro.2017.10 .182

\begin{tabular}{l}
\hline Lilian Keylla Berto \\
Acadêmica de Engenharia Civil doCentro \\
Universitário de Maringá - Unicesumar. Bolsista \\
PROBIC/UniCesumar. \\
\hline
\end{tabular}

\section{RenanGodoi}

Engenheiro Civil, formado no Centro Universitário de Maringá - Unicesumar.

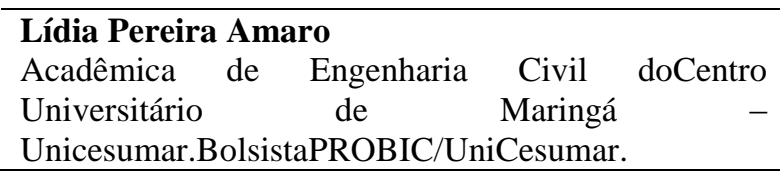

\begin{tabular}{l}
\hline Judson Ricardo Ribeiro \\
Engenheiro Civil. Mestre em Engenharia de \\
Materiais.Coordenador e professor no Centro \\
Universitário de Maringá - Unicesumar. \\
\hline
\end{tabular}

Luciana Cristina Soto Herek Rezende

$\mathrm{PhD}$, Pesquisadora do Instituto Cesumar de Ciência, Tecnologia e Inovação - ICETI, Unicesumar. 